\title{
New Results on Quasar Outflows
}

\author{
Fred Hamann ${ }^{1}$, Nissem Kanekar, Jason X. Prochaska, \\ Michael T. Murphy, Nikola Milutinovic, Sara Ellison, \\ Wim Ubachs, and Gary Ferland \\ ${ }^{1}$ University of Florida, Department of Astronomy, Gainesville, FL 32611-2055, USA
}

\begin{abstract}
Accretion disk outflows are an important part of the quasar phenomenon. They might play a major role in distributing metals to the galactic surroundings, halting growth of the central black hole and providing kinetic energy "feedback" to regulate star formation in the host galaxies. Some models of galaxy evolution indicate that feedback requires kinetic energy luminosities, $L_{\mathrm{K}}$, that are $\sim 5 \%$ of the quasar bolometric; $L_{\mathrm{K}} / L=\dot{M}_{\mathrm{w}} v^{2} / 2 \eta \dot{M}_{\mathrm{acc}} c^{2} \sim 5 \%$ is possible if $\dot{M}_{\mathrm{w}} \sim \dot{M}_{\text {acc }}$ (with $v \sim 0.1 c$, and $\eta \sim 0.1$ ). Here we describe results from two studies designed to test the theoretical energetics of radiatively driven outflows and derive observational constraints on the outflow geometry and physical properties emphasizing weaker outflow features like NALs and mini-BALs.
\end{abstract}

Keywords. quasars: general, quasars: absorption lines, galaxies: evolution, line: formation

High-Velocity NALs: We report on the discovery of variable, high-velocity narrow absorption lines (NALs) in the $z=2.26$ quasar J 2321-0050. Five distinct systems in the UV lines of C IV and OVI have $v \sim 8200-12500 \mathrm{~km} \mathrm{~s}^{-1}, \mathrm{FWHM} \sim 62-164 \mathrm{~km} \mathrm{~s}^{-1}$, metallicity $\sim 2 Z_{\odot}$, and total column density of a few times $10^{19} \mathrm{~cm}^{-2}$. The line strength variations are coordinated between the systems and appear to be controlled by changes in the line-of-sight covering fractions. The data require a complex outflow geometry with five distinct absorbing regions that are each inhomogeneous and smaller than the UV continuum source: $\sim 0.04 \mathrm{pc}$ in $\mathrm{CIV}$ and $\sim 0.05-0.06 \mathrm{pc}$ in O VI. An upper limit on the acceleration, $<3 \mathrm{~km} \mathrm{~s}^{-1} \mathrm{yr}^{-1}$, is consistent with blobs moving near the terminal speeds at distances $>5 \mathrm{pc}$ from the central quasar. An apparent double line-lock in C IV suggests the flow was radiatively acceresent trajectory is within $16^{\circ}$ of the radial direction. The coordinated nature of the line variations and the short time scales $(<0.63 \mathrm{yr}$ in the quasar frame) are best explained by global ionization changes induced by changes in the quasar's ionizing flux.

Outflow Models: We are testing basic ideas about quasar outflows using the numerical code CLOUDY, which iterates to solve the equations of motion and mass continuity with self-consistent treatments of the temperature, ionization, excitation, opacities, and (importantly) the line overlap caused by velocity shifts in a moving medium. The radiative force is determined precisely from the line+continuum opacities and the transmitted spectrum at each location in the flow. The simple 1-D geometry allows us to examine a wide range of circumstances (launch radius, density at launch, metallicity, $L / L_{\mathrm{Edd}}$, incident spectral shape with or without a separate X-ray/far-UV shielding medium, etc.) and thereby determine what ranges of flow speeds, column densities, mass loss rates, and kinetic energy yields are possible with radiative driving. Early results suggest that, with the right initial conditions, flows driven by radiative forces can indeed reach speeds of order $0.1 c$ with $L_{\mathrm{K}}$ yields high enough to be important for feedback to galaxy evolution. 